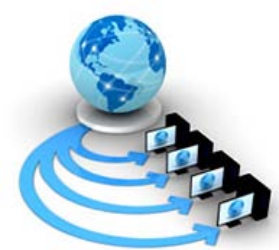

Volume 8, No. 8, September-October 2017

International Journal of Advanced Research in Computer Science

RESEARCH PAPER

Available Online at www.ijarcs.info

\title{
GLC AND GLC** CONTINUOUS FUNCTIONS: A CONCEPTUAL FLAW
}

\author{
P.L. Powar \\ Department of Mathematics and Computer Science \\ R.D. University, Jabalpur,India
}

\author{
Pratibha Dubey \\ Department of Mathematics, St. Aloysius College, \\ Jabalpur,India
}

\begin{abstract}
The concept of generalized locally closed sets (glc-sets), GLC**-sets followed by the notion of GLC and GLC**continuous maps was initiated by Balachandran et al. (Generalized locally closed sets and GLC-continuous functions, Indian J. pure appl. Math 27(3): 235-244, 1996). In the present work, it has been established that the collection of glc-sets and the collection of GLC** -sets, each is exactly equal to the power set $\mathrm{P}(\mathrm{X})$ of X. Consequently, any arbitrary function with any choice of domain and range turns out to be GLC and GLC**-continuous function which is not desirable from analytic point of view.
\end{abstract}

Keywords: Topological spaces, locally closed sets, glc-set, GLC **-set, GLC-continuity, GLC**-continuity. AMS Subject Classification (2000): primary 54XX; secondary 54CXX.

\section{INTRODUCTION}

The idea of locally closed set was introduced by Bourbaki [2] in 1966. (see also [3]). This concept of locally closed set had been used by Ganster and Reilly [4] for defining the generalized version of continuity viz. LC-irresolute, LCcontinuity and sub-LC-continuity. Balachandran et al. [1] had extended the definition of locally closed sets and initiated the notion of "Generalized locally closed set", in particular, glcset, GLC*-set and GLC**-set. Since last few decades many topologist (cf. [4], [5], [6], [7], [8], [9], [10], [11]) are trying to explore the possibility of generalizing the classical phenomenon "continuity" of the function defined in the topological space. Following this trend Balachandran et al. [1] have also defined and explored the idea of GLC-irresolute maps and GLC-continuous maps. Extending the idea of Balachandran et al. [1], Park et al. [8] have defined semi generalized locally closed sets and locally-generalized closed sets along with SGLC-continuous functions and L $\delta$ GLCcontinuous functions respectively. (see also [9], [10], [11]).

Recently, Patil et al. [10] have further extended the concept of glc-sets and introduced the notion of

$\mathrm{g}^{*} \mathrm{w} \alpha$-lc sets and $\mathrm{g}^{*} \mathrm{w} \alpha^{*}$-lc sets and $\mathrm{g}^{*} \mathrm{w} \alpha^{* *}$-lc sets and have applied these concepts to define relevant different types of continuous functions.

In the present paper, authors have established that the respective collection of glc-sets, and the collection of GLC**sets (cf. [1]) generated by the topology yield precisely the power set $\mathrm{P}(\mathrm{X})$ of $\mathrm{X}$. This information leads to the conclusion that the corresponding GLC and GLC** - idea of continuity is not enhancing the class of continuous functions with some relaxed conditions however all functions with arbitrary domain and range turns out to be GLC and GLC**-continuous functions which is inadequate. In view of this observation, all the extensions turned out to be superfluous.

\section{PRE-REQUISITES}

The following notations have been referred throughout this work:

$(\mathrm{X}, \tau)$ - $\quad$ Topological space with topology defined on

$\operatorname{cl}(\mathrm{A})$ - $\quad$ the set $\mathrm{X}(\neq \phi)$. respect to $(\mathrm{X}, \tau)$.

$\operatorname{int}(\mathrm{A})-\quad$ Interior of $\mathrm{A}$ for the subset $\mathrm{A}$ of $\mathrm{X}$ with respect to $(\mathrm{X}, \tau)$.

$\mathrm{P}(\mathrm{X})$ - $\quad$ Power set of $\mathrm{X}$.

Definition 2.1. A subset B of (X, $\tau)$ is called g-closed [12] if $c l(B) \subseteq G$ whenever $B \subseteq G$ for an open set $G$ in a topological space $(\mathrm{X}, \tau)$. A subset $C$ of $(\mathrm{X}, \tau)$ is called g-open if its complement $X-C$ is g-closed.

Example 2.1. Consider a topological space $X=\{a, b, c, d\}$ with the topology $\tau=\{\mathrm{X}, \phi,\{\mathrm{a}, \mathrm{b}, \mathrm{c}\}\}, \mathrm{F}_{\mathrm{X}}=\{\mathrm{X}, \phi,\{\mathrm{d}\}\}$, where $\mathrm{F}_{\mathrm{X}}$ is the collection of closed sets in $(\mathrm{X}, \tau)$. Let $\mathrm{A}=\{\mathrm{a}$, d) be a subset of $X$. There is only one open set say $U=X$ containing $\mathrm{A}$. Then it is easy to check that $c l\{\mathrm{a}, \mathrm{d}\}=\mathrm{X}$ which follows by the definition that $c l\{\mathrm{a}, \mathrm{d}\}=\mathrm{X}=\mathrm{U}=\mathrm{X}$. Hence $\mathrm{A}$ $=\{\mathrm{a}, \mathrm{d}\}$ is g-closed.

Remark 2.1. It is a direct consequence from the definition of g-closed sets that every open set is g-open and every closed set is g-closed but the respective converse is not true in general.

Definition 2.2. Let $S$ be a subset of a topological space $(X, \tau)$. $S$ is said to be generalized locally closed (glc-set) [1] if there exists g-open set $G$ and g-closed set $F$ such that $S=G \cap F$. The collection

of all generalized locally closed set is denoted by GLC (cf. [1]). 
Example 2.2. Consider a topological space $X=\{a, b, c, d\}$ with the topology $\tau=\{\mathrm{X}, \phi,\{\mathrm{b}, \mathrm{c}, \mathrm{d}\},\{\mathrm{a}, \mathrm{c}, \mathrm{d}\},\{\mathrm{c}, \mathrm{d}\}\}, \mathrm{F}_{\mathrm{X}}=$ $\{\tau, \mathrm{X},\{\mathrm{a}\},\{\mathrm{b}\},\{\mathrm{a}, \mathrm{b}\}\}$. In view of Definition 2.1 , the collection of g-closed sets $=\{X, \phi,\{a\},\{b\},\{a, b\},\{a, b, c\}$, $\{a, b, d\}\}$ and the collection of g-open sets $=\{\phi, X,\{b, c, d\}$, $\{a, c, d\},\{b, c, d\},\{a, c, d\},\{c, d\},\{d\},\{c\}\}$. We now show that $\mathrm{A}=\{\mathrm{b}, \mathrm{c}\} \subseteq \mathrm{X}$ is a glc-set.

Claim: $A=\{U \cap V$ : $U$ is g-open and $V$ is g-closed $\}$.

We now consider $U=\{b, c, d\}$ a g-open set and $V=\{a, b$, c) a g-closed set. Then,

$\mathrm{U} \cap \mathrm{V}=\{\mathrm{b}, \mathrm{c}, \mathrm{d}\} \cap\{\mathrm{a}, \mathrm{b}, \mathrm{c}\}=\{\mathrm{b}, \mathrm{c}\}$ is a glc-set. It may be verified easily that the collection of all glc-sets is exactly equal to $\mathrm{P}(\mathrm{X})$.

Remark 2.2. It is clear that every g-closed set is glc-set and every g-open set is glc-set.

Definition 2.3. Consider a subset $S$ of a topological space. Then $S \in$ GLC** $^{*}$ if $S \in G \cap F$ for any open set $G$ and a gclosed set $\mathrm{F}$ of $(\mathrm{X}, \tau)$ respectively (cf.[1]).

Definition 2.4. Let (X, $\tau$ ) and $(\mathrm{Y}, \sigma)$ be two topological spaces. A function $f: \rightarrow(\mathrm{X}, \tau) \rightarrow(\mathrm{Y}, \sigma)$ is said to be GLCcontinuous (resp. GLC**-continuous) if $f^{-1}(\mathrm{~V}) \in$ GLC (resp. $f^{-1}(\mathrm{~V}) \in \mathrm{GLC}^{* *}$ ) for each $\mathrm{V} \in \sigma$ (cf.[1]).

Definition 2.5. A function : $(\mathrm{X}, \tau) \rightarrow(\mathrm{Y}, \sigma)$ is said to be GLC-irresolute (resp. GLC**-irresolute) if $f^{-1}(V) \in \mathrm{GLC}$ (resp. $f^{-1}(V) \in \mathrm{GLC}$ ) for each $\mathrm{V} \in \mathrm{GLC}$ (resp. $\mathrm{V} \in \mathrm{GLC} * *$ ) in $(\mathrm{Y}, \sigma)$ (cf.[1]).

Claim:

\section{MAIN RESULT}

We are now set to state the main result of this paper.

Theorem 3.1. Let $(\mathrm{X}, \tau)$ be the topological space and GLC and GLC** be the collection of sets described in the Definition 2.2 and 2.3 respectively. Then

$\mathrm{GLC} \cong \mathrm{GLC}^{* *} \cong \mathbf{P}(\mathrm{X})$

where $\mathrm{P}(\mathrm{X})$ is the power set of $\mathrm{X}$.

Proof. Let $\mathrm{X}$ be any non empty set $\tau=\left\{\phi, \mathrm{X},\left\{\mathrm{U}_{\alpha}\right\}_{\alpha \in J}\right\}$ be the topology on $\mathrm{X}$. Let $\mathrm{A}$ be any non empty proper subset of $\mathrm{X}$. The following cases have been considered:

Case 1. A $\nsubseteq U_{\alpha}(\neq \mathrm{X})$ for all $\alpha \in \mathrm{J}$ and $U_{\alpha} \in \tau$ implies $\mathrm{A}$ $\subseteq \mathrm{X}$ only. It is clear that $c l(\mathrm{~A}) \subseteq \mathrm{X}$. Hence, $\mathrm{A}$ is g-closed. Referring Remark 2.2, we conclude that $\mathrm{A}$ is glc-set.

Case 2. $\mathrm{A} \subseteq U_{\alpha}$ for some $\alpha \in \mathrm{J}$ and CA $\subseteq \mathrm{X}$ but $\mathrm{CA} \nsubseteq U_{\alpha}$ for each $\alpha \in \mathrm{J}$ where $\mathrm{C}$ stands for the complement of $\mathrm{A}$ in $\mathrm{X}$. It is obvious that $\operatorname{cl}(\mathrm{CA}) \subseteq \mathrm{X}$. Hence CA is gclosed which implies that $\mathrm{A}$ is g-open. In view of Remark 2.2, the set $\mathrm{A}$ is glc again.

Case 3. $\mathrm{A} \subseteq U_{\alpha}$ for some $\alpha \in \mathrm{J}$ and CA $\subseteq U_{\delta}$ for some $\delta \in \mathrm{J}$ where $U_{\alpha}, U_{\delta} \in \tau$. Let if possible that $\mathrm{A}$ is neither $\mathrm{g}$ open nor g-closed.

$$
A=G_{c} \cap G_{o}
$$

where $\mathrm{G}_{\mathrm{c}}$ and $\mathrm{G}_{\mathrm{o}}$ are g-closed and g-open sets in $(\mathrm{X}, \tau)$ respectively. Since, $\mathrm{A}$ is not g-closed, there exists at least one index $\beta \in \mathrm{J}$ such that

$$
\mathrm{A} \subseteq U_{\beta} \text { but } \operatorname{cl}(\mathrm{A}) \nsubseteq U_{\beta} \text { (cf. Definition 2.1) Then }
$$

Either

$$
D(A) \subseteq C U_{\beta}
$$

Or

$$
\text { (b) } S(\neq \phi) \subseteq D(A) \text { and } S \subseteq C U_{\beta} \text { such that } \mathrm{A} \cup \mathrm{D}(\mathrm{A})_{\sim S} \subseteq U_{\beta}
$$

Consider the set $A \cup C U_{\beta}$

Claim: $A \cup C U_{\beta}$ is g-closed.

There exists a family $\left\{U_{\alpha}^{*}\right\}_{\alpha \in J}$ of open sets such that

$$
A \cup C U_{\beta} \subseteq U_{\alpha}^{*} \quad \text { for } \alpha \in \mathrm{J}
$$


Since $A \subseteq A \cup C U_{\beta}$, the collection $\left\{U_{\alpha}^{*}\right\}_{\alpha \in J}$ is a sub-collection of $\left\{U_{\alpha}\right\}_{\alpha \in J}$ of open sets containing A.

i) $\quad$ Consider $\beta \in \mathrm{J}$ such that $A \cup C U_{\beta} \subseteq U_{\beta}^{*}$ and $U_{\beta} \subseteq U_{\beta}^{*}$.

- If $U_{\beta}^{*}=X$, then $\operatorname{cl}\left(A \cup C U_{\beta}\right) \subseteq X$ and $A \cup C U_{\beta}$ is g-closed.

- If $U_{\beta}^{*}(\neq \phi, X)$, then

$$
\begin{aligned}
\operatorname{cl}\left(A \cup C U_{\beta}\right) & =\operatorname{cl}(A) \cup \operatorname{cl}\left(C U_{\beta}\right) \\
& =A \cup D(A)_{\sim} s \cup S \cup C U_{\beta}\left(\because C U_{\beta} \text { is closed }\right) \\
& =A \cup D(A)_{\sim} s \cup C U_{\beta} \\
& \subseteq U_{\beta}^{*}\left(\because C U_{\beta} \subseteq U_{\beta}^{*} ; U_{\beta} \subseteq U_{\beta}^{*}\right. \text { and using (3.3)) }
\end{aligned}
$$

Therefore $A \cup C U_{\beta}$ is g-closed.

ii) We next consider $\alpha(\neq \beta) \in \mathrm{J}$ such that $A \cup C U_{\beta} \subseteq U_{\alpha}^{*}$ for $U_{\alpha} \subseteq U_{\alpha}^{*}$ and $\operatorname{cl}(A) \subseteq U_{\alpha}$

$$
\begin{array}{llc}
\operatorname{cl}\left(A \cup C U_{\beta}\right) & & \operatorname{cl}(A) \cup \operatorname{cl}\left(C U_{\beta}\right) \\
& = & \operatorname{cl}(A) \cup C U_{\beta} \\
& \subseteq & U_{\alpha}^{*}
\end{array}
$$

Thus, $A \cup C U_{\beta}$ is g-closed.

Claim: $A=\left(A \cup C U_{\beta}\right) \cap U_{\beta}$ where $G_{c}=A \cup C U_{\beta}$ is g-closed and $G_{o}=U_{\beta}$ is g-open (cf. Remark 2.1). Consider

$$
\begin{aligned}
G_{C} \cap G_{o} & =\left(A \cup C U_{\beta}\right) \cap U_{\beta} \\
& =\left(A \cap U_{\beta}\right) \cup\left(C U_{\beta} \cap U_{\beta}\right) \\
& =A
\end{aligned}
$$

Hence, (3.1) holds and finally we conclude that A is glc-set. Since, A was arbitrary subset of X, every subset of X is glc-set. Thus, the collection GLC is precisely equal to $\mathbf{P}(\mathbf{X})$.

Since $G_{o}=U_{\beta}$ (open), it is direct by the Definition 2.3 that

This completes the proof.

$$
\mathrm{GLC}^{* *} \cong \mathbf{P}(\mathrm{X})
$$

\section{CONCLUSION}

- In view of Definitions 2.4 and 2.5, each function $f$ defined from $(\mathrm{X}, \tau)$ to $(\mathrm{Y}, \sigma)$ turns out to be GLCcontinuous (irresolute) and GLC** -continuous (irresolute) which is not acceptable as a generalization of the classical concept of continuity in Topology.

\section{REFERENCES}

- All generalizations of GLC-set and GLC** -set turned out to be stagnant and finally not desirable. 
[1] K. Balachandran, P. Sundaram, H. Maki, "Generalized locally closed sets and GLC-continuous functions", Indian J. pure appl. Math., vol. 27(3), pp. 235-244, March 1966.

[2] N. Bourbaki, "General Topology", Part I., Addition Wesley, Reading Mass, 1966.

[3] A. H. Stone, "Absolutely FG spaces”, Proc. Amer. Math. Soc. vol. 80, pp. 515-520, 1980.

[4] M. Ganster, L. Reilly, M.K. Vamanamurty, "Locally closed sets and LC-continuous functions," Int. Jl. Math. Soc., vol. 12, pp. 417- 424, 1989.

[5] S.S. Benchalli, P.G. Patil, T.D. Rayanagaudar, "wclosed sets in topological spaces", The Global. J. Appl. Math. Sci., vol. 2, pp. 53-63, 2009.

[6] S.S. Benchalli, P.G. Patil, P.M. Nalwad," Generalized w-closed sets in topological spaces", Journal of New Results in Science, ISSN 1304-7981, Number 7, pp.7-19, 2014.

[7] E. Ekici, "On locally closedness and continuity", Chaos, Solitons and Fractals, vol. 36, pp. $1244-1255$, 2008.
[8] J.H. Park, J.K. Park, "On semi generalized locally closed sets and SGLC-continuous functions”, Indian J. pure appl. Math., vol. 31(9), pp. 1103-1112, Sept. 2000.

[9] J.H. Park, J.K. Park, “On locally $\delta$-generalized closed sets and L $\delta$ GLC-continuous functions", Chaos, Solitons and Fractals, vol. 19(4), pp. 995 -1002, 2004 (Elsevier).

[10] P.G. Patil, S.S. Benchalli, P.S. Mirajkar, "Decomposition of locally closed sets in topological spaces”, Advances in Fuzzy Mathematics (AFM), vol. 12, No. 1, pp. 101-110, 2017.

[11] K. Chandrashekhar Rao, K. Kannan, "Some properties of g-locally closed sets", Journal of Advanced Research in Pure Mathematics, vol.1, No. 1, pp. 1-9, 2009.

[12] N. Levine, "Generalized closed sets in topology", Rent. Circ. Mat. Palermo, vol. 19(2), pp. 89-96, 1970. 